\title{
Estimation of Fit in Calves for Supporting Internet Boot Sales
}

\author{
Damir OMRČEN*, Tina VIDIĆ* \\ UCS - Universal Customization System, Vrhnika, Slovenia
}

\begin{abstract}
The paper introduces a system for estimating the fit of boots in calves. The system recommends a better fitting boot. Such a system is an essential component for Internet boot sales. The key module of the system is a device for inner circumference measurement of boot shafts. The boot measurement in combination with customer's calf measurement defines the fit of boot. The system successfully estimates the fit and recommends a good fitting boot for a customer when buying boots online.

We have performed a detailed survey with 93 female subjects. The subjects have evaluated the fit in calves of 38 boot models. The data has been later used as training data for a sophisticated system for fit estimation and recommendation.

The boot recommendation system in combination with UCS lower shoe recommendation is a perfect solution for an online retailer.

The paper describes the measurement device, the survey and the estimation algorithm.
\end{abstract}

Keywords: calf girth measurement, boot shaft circumference measurement, estimation of boot fit in calves, shoe recommendation system

\section{Introduction}

Calf girths in females are very heterogeneous. In 1988 US Army performed a measurement campaign of 2208 female subjects in ages of 18-50 years [1]. The campaign has shown that the girth in calves ranges from $285 \mathrm{~mm}$ to $459 \mathrm{~mm}$, with notation that dispersion in population is even greater. The study did not show any bigger correlation between foot length or width to the calf girth, so it is difficult to use the foot measurement for calf fit estimation.

The boot producers has adapted to the dispersion of the population and produce boots in various widths. Due to very diverse girths in calves and on the other hand very diverse widths of boots it is very difficult for a person to buy boots - especially online without the possibility to put on and to estimate the fit of a boot. The actual circumstances in the market show big importance of that problem. Also the survey presented in this work confirms this statement.

To overcome this problem some sellers (and/or producers) label their products depending on the circumference of the shaft - for example Wide, ExtraWide, XXL, shaft $40 \mathrm{~cm}$ wide etc. For examples see [2-5]. Some retailers present the rules for selecting proper boot in calves based on customer's calf measurement. The rules are usually as the following: if your foot size is $9 \mathrm{E}$ and your calf is $454 \mathrm{~mm}$ than you should order Extra Wide boot. For example see [5].

There are many problems with the existing solutions: Many producers do not label their boots. Standards for shaft width are not very well defined. Some shafts are elastic, some not. Sometimes a boot fits well to a narrow leg as well as to a wide leg. Some customers also don't know their calf size or they do not know how to use complex rules for getting the right fitting boot. Due to these facts we propose an advanced system that estimates the fit of boots based on the customer's measurements and boot database. By that a customer does not need to guess if a boot fits to her leg, the system does it for her - with a high success rate. Such system was also initiated by the requirements from the market.

In this document we describe the whole system for estimation of calf fit. The system called BoC (stands for Boot Calf) estimates the fit of boots depending on the customer's calf measurements and boot measurements. In the document we describe the device for boot measurement, the measurement campaign and the analysis of results, and introduce a fit estimation algorithm.

The product can work as an independent solution or even better in combination with UCS shoe size and fit recommendation system (presented at 3D body scanning conf. 2011 [6]).

\section{Methods}

The UCS BoC system is based on accurate boot shaft measurement and measurement of person's calf girth. The final result of the system is the estimation of fit in calf for a specific person and boot. This result can be used on a web page as boot recommendation or for filtering boot selection.

\footnotetext{
*damir.omrcen@ucstech.eu; tina.vidic@ucstech.eu
} 
The UCS BoC fit estimation system works in the following way (see also Fig. 1):

- Boot shaft circumference measurement

All boot models are measured simply and effectively with UCS BoC measurement device. It is a patented system for inner boot shaft circumference measurement.

- Measurement of calf girth

Customer measures the calves girth at home simply by using a tape measure.

- Calf fit estimation

Fit in calves is estimated considering customer's calves' measure and boot measurement. Additionally, the system considers elasticity of the material, boot type and customer's personal preferences.

- Fit estimation is expressed in multiple levels: e.g. too narrow, perfect fit, too wide

- Finally, UCS BoC estimation is combined with lower part of shoe recommendation

In case of bad fit a better boot can be recommended. The complete solution results in a satisfied customer and decreases the return rate of Internet sales.

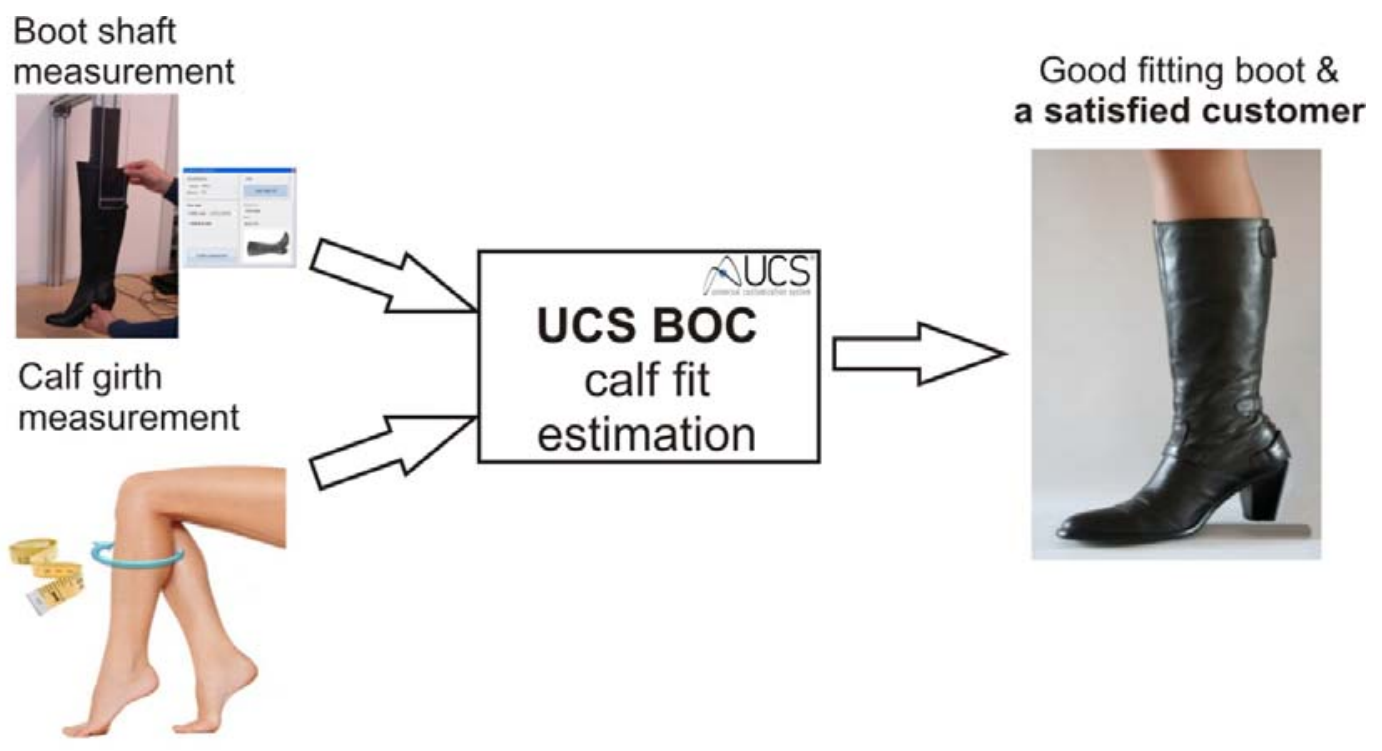

Fig. 1. Schematics of UCS BOC fit estimation system

\subsection{BoC measurement device}

The key component of BoC system is the device for inner boot circumference measurement. The device is designed for mass measurement of boots. It is therefore robust, simple and industrial design (see Fig. 2). It takes less than 15 seconds to measure a boot.

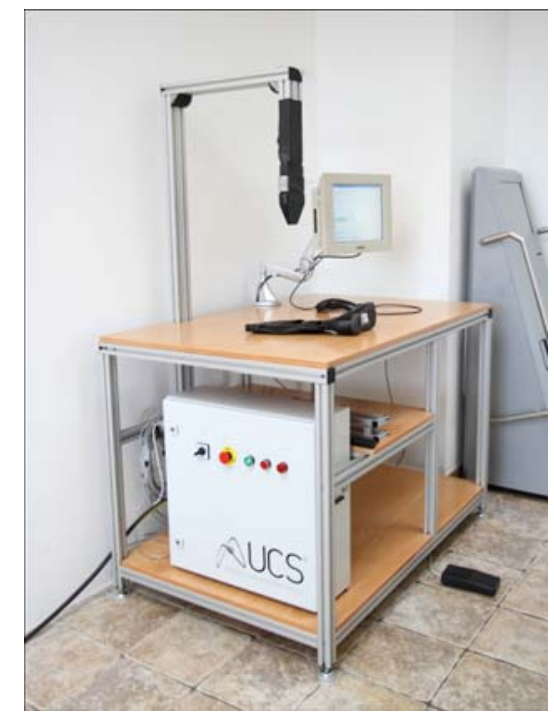

Fig. 2. BoC measurement device 
The measurement unit shown in Fig. 3 is composed of pneumatic actuators that expand and apply suitable pressure inside of a boot. The pressure is similar to the pressure normally being present when a boot is on a leg. Positional sensors measure the extension of the pneumatic actuators and define the circumference. Using this device we are able to measure one or multiple circumferences along the boot shaft.
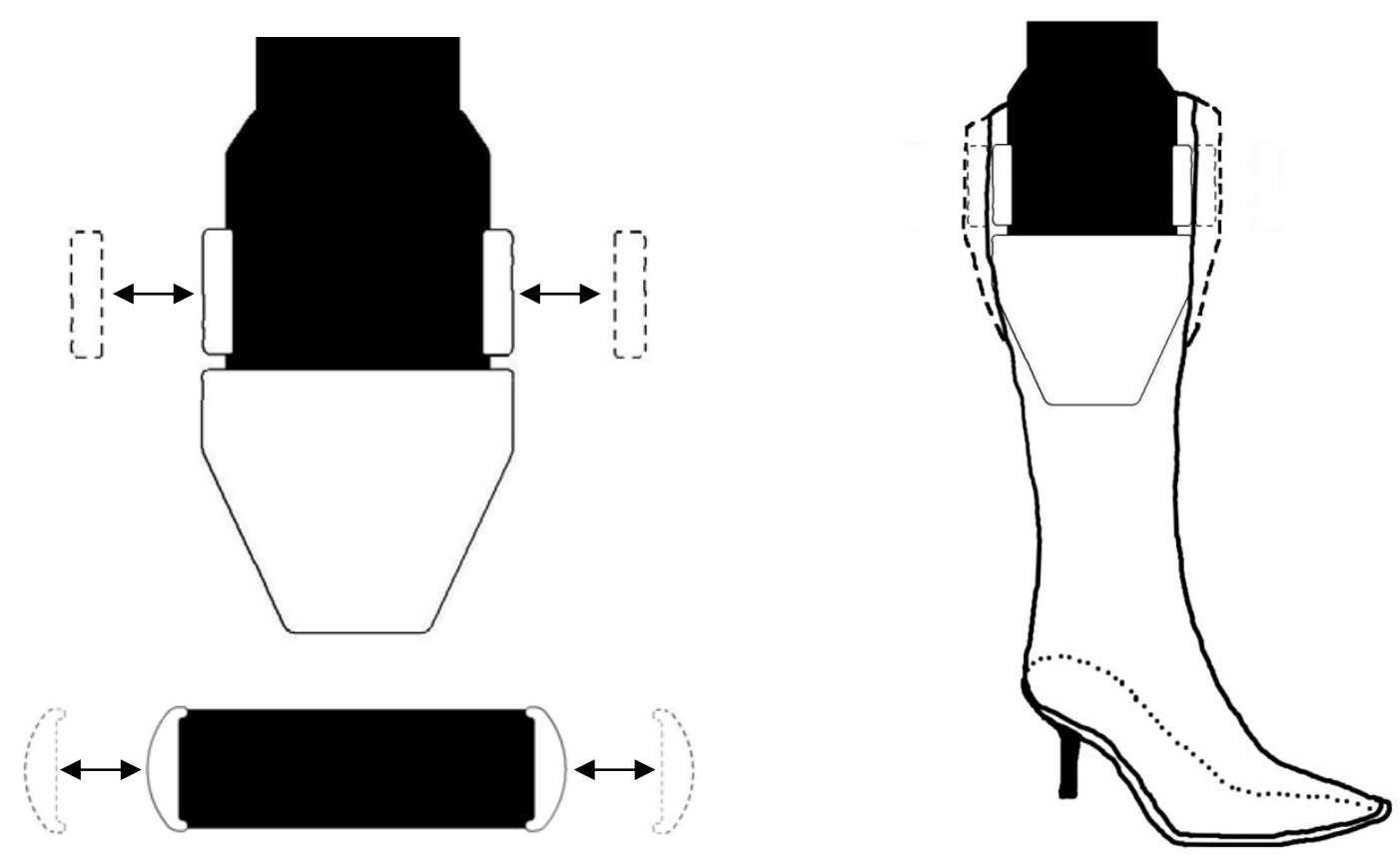

Fig. 3. BoC measurement unit detail

\subsection{The survey}

93 female subjects participated in the survey. The subjects' age was from 17 to 65 years. The weight distribution was similar to the normal population. The subjects tested 38 different boot models from different boot styles (tight, loose, low, high, stretchy, fashion, comfort, etc.). The subject's calves were measured precisely, as well as the boots.

The subjects were asked to put on the boots and evaluate the fit of boot in the calf region. They were asked to ignore the fit in the lower part of the boot, to ignore the color, style etc. Only the fit in calves was of interest.

The subject could estimate the fit in calves in the following levels:

\begin{tabular}{|r|l|}
\hline-2 & Boot shaft is too narrow - unacceptably narrow (would not buy it) \\
\hline-1 & Boot shaft is slightly too narrow - but is acceptable (would buy it) \\
\hline 0 & Perfect fitting boot \\
\hline+1 & Boot shaft is slightly too wide - but is acceptable (would buy it) \\
\hline+2 & Boot shaft is too wide - unacceptably wide (would not buy it) \\
\hline
\end{tabular}

All the subjects were asked to measure girths of both calves at various heights using a tape measure. The girths were measured in sitting position by having both legs relaxed.

\subsection{Fit estimation algorithm}

Based on the boot measurement and results of questionnaires a precise algorithm that estimates the fit of boot for a person have been developed.

We use basic artificial intelligence approaches to define fit estimation model [7]. We set-up and train a fit estimation model using a set of training data. The training data are pairs of input data and target data. The input data are calf and boot circumferences, while the target data are the fit estimation obtained from the subjects (see Fig. 4). It is very important that the training data cover the whole range of expected inputs so that it generalizes well within the range of inputs for which they have been trained. 


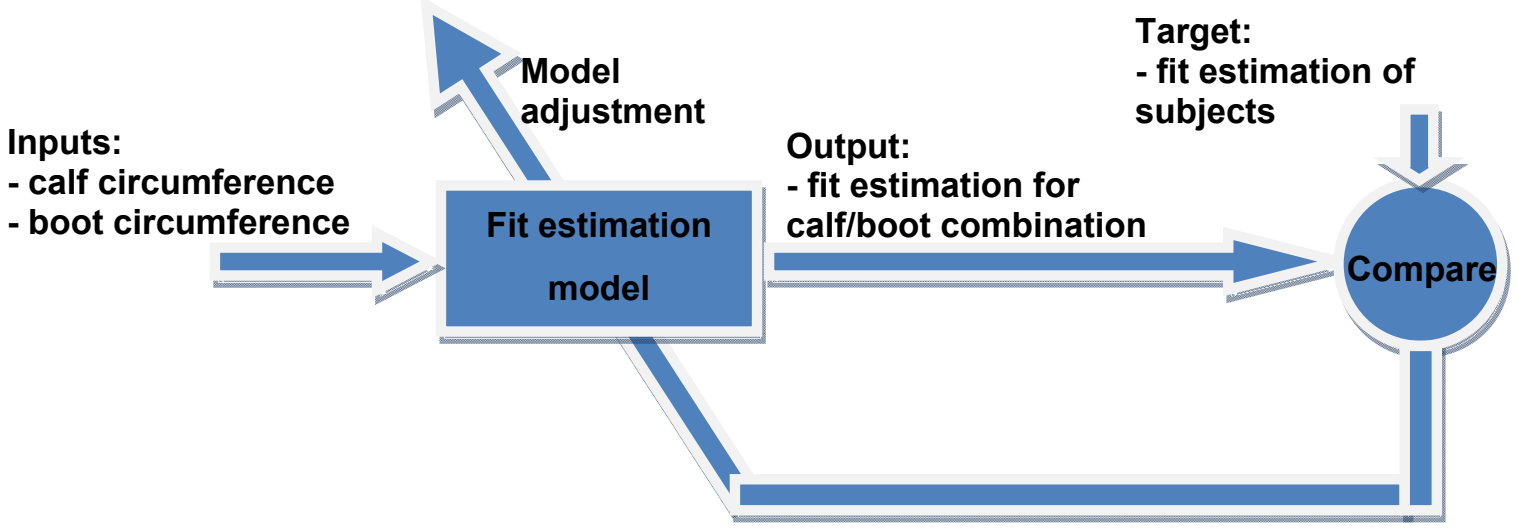

Fig. 4. Training (setting up) the fit estimation model

Training of the model is done in the following way. First we define the structure of the model. The model can be a simple function (linear, quadratic, polynomial...), neural network model, SVM model etc. Then training is executed, where the parameters of the model are adjusted, or trained, so that a particular input leads to a specific target output. In the Fig. 4 the model is adjusted based on the comparison of the model output and the target until the model output matches the target. Typically, many such input/target pairs are needed to train a model.

\section{Results}

\subsection{Overview of training data}

\subsubsection{Boot measurement}

We have measured 38 boot models in all available sizes. The grading (or production accuracy) is rather poor. Graphs in Fig. 5 show the circumferences of boots versus boot size. We would expect that the circumference is monotonically increasing (or is even a linear) function. Fig. 5a shows an example of a good graded boot model, while Fig. $5 \mathrm{~b}$ shows a badly graded model.

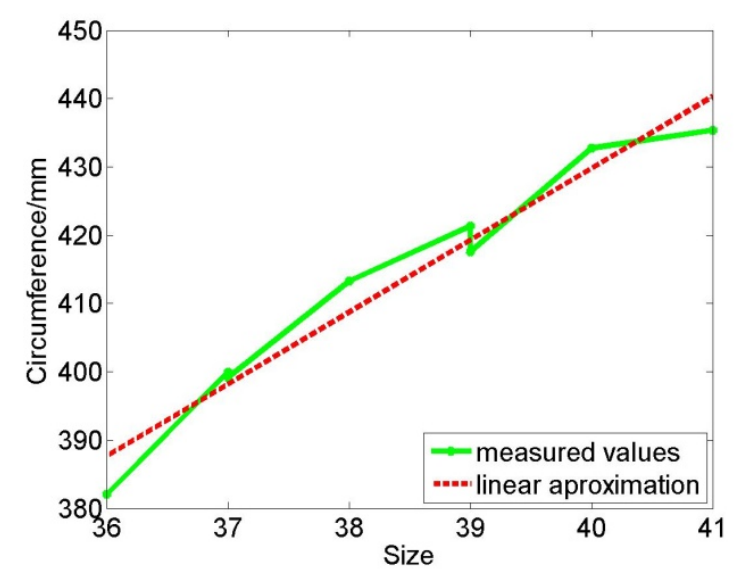

a. Well graded model

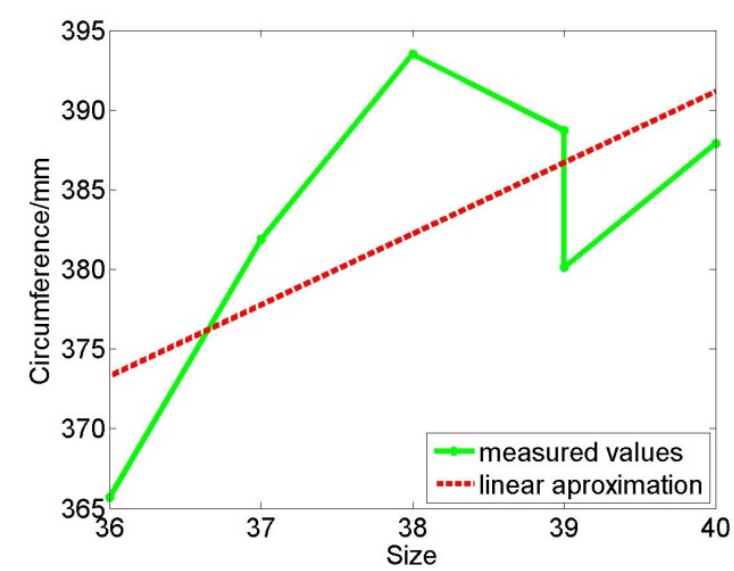

b. Badly graded model

Fig. 5. Examples of boot shaft grading

We can see in Fig. 5 b that also for the same size shoe (size 39) different measurements exist - some boots are narrower than the other of the same model and size. To make the measurements useful for the recommendation system we approximate the data as shown with the red dashed lines in Fig. 5.

Fig. 6 shows linear approximation of circumference of different boot models. We can see that some models are wider, some are narrower, and some are in between. Grading is not constant between models, so there are models that are very narrow at small sizes, but are medium wide at bigger sizes. Therefore, it would be unfair to classify the whole model into one group. 


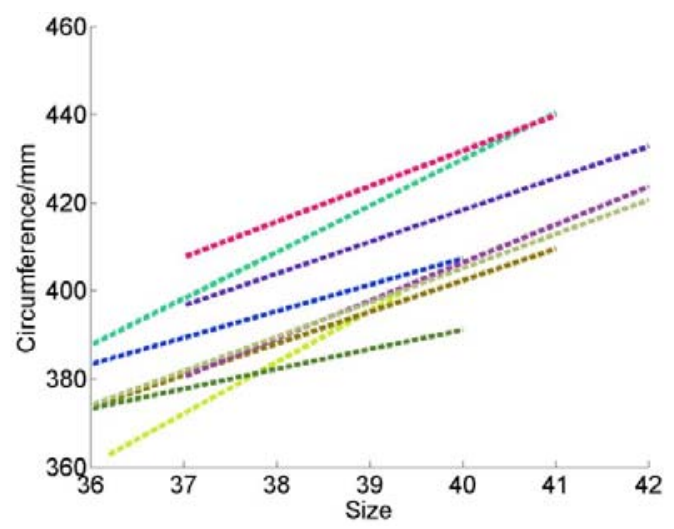

Fig. 6. Comparison of grading of some boots models

The boot collection in the survey is rather low, even though the variety of styles and widths is a good representative of the boots available in the market. Additionally, the model for fit estimation must be designed in the way that it generalizes well even outside of the training data.

\subsubsection{Subject measurements}

Subjects have measured their calves using a tape measure. The subjects should cover general population in order to provide good estimation for a random customer. Here, we analyze only the maximal girth of calf. The next table shows the characteristic data of the test group.

\begin{tabular}{|l|l|l|l|}
\hline Min. calf girth & Max. calf girth & Mean calf girth & Std. dev. of calf girth \\
\hline $314 \mathrm{~mm}$ & $453 \mathrm{~mm}$ & $374 \mathrm{~mm}$ & $33.4 \mathrm{~mm}$ \\
\hline
\end{tabular}

The test group does not cover the whole population (comparing to [1]), however, we believe that it is a good representative of the population.

\subsubsection{The survey}

The subjects have evaluated the fit of calf. The evaluation should be properly distributed for good model training. For example, we cannot train the model well, if none of the evaluation is too narrow $(-2)$. The next table shows the distribution of evaluations:

\begin{tabular}{|l|c|c|c|c|c|}
\hline evaluation & -2 & -1 & 0 & 1 & 2 \\
\hline part in \% & 19.4 & 5.6 & 43.8 & 13.8 & 17.4 \\
\hline
\end{tabular}

The evaluation is well distributed.

\subsection{Fit estimation model}

Fit estimation model should estimate the fit depending on input parameters (calf girth and boot circumference). Based on its estimation proper boots are offered to a customer.

The quality of fit estimation or the accuracy of estimation $e s t_{a c c}$ is defined by the number of correct estimations by the following equation:

$$
\text { est }_{\text {acc }}=\frac{N_{\text {est }==1}}{N_{\text {all }}}
$$

where $N_{\text {est }==1}$ and $N_{\text {all }}$ are the number of correct estimations and the number of all instances, respectively.

Each instance counts as correct (1) if the model estimation is "the same" as the subject's evaluation. We define the correctness of estimation as:

$$
\text { est }=\left\{\begin{array}{cc}
\left(\text { model }_{\text {est }} \in\{-2\}\right) \&\left(\text { subject }_{\text {eval }} \in\{-2\}\right) \mid \\
1, \text { if } & \left(\text { model }_{\text {est }} \in\{-1,0,1\}\right) \&\left(\text { subject }_{\text {eval }} \in\{-1,0,1\}\right) \mid \\
& \left(\text { model }_{\text {est }} \in\{2\}\right) \&\left(\text { subject }_{\text {eval }} \in\{2\}\right) \\
0, \text { if } & \text { otherwise }
\end{array}\right.
$$

where model $_{\text {est }}$ is the model estimation and subject $_{\text {eval }}$ is the subject's evaluation. The equation above does not distinguish between values $-1,0$ and +1 , since all values indicate "boot is acceptable". 
The fit estimation model is defined with the following model:

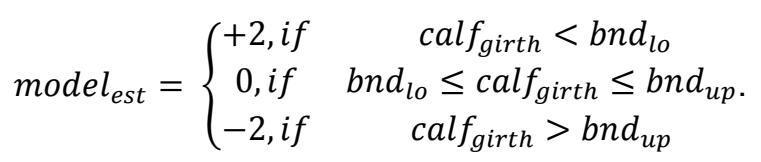

Here, calf $f_{\text {girth }}$ is the girth of subject's calf, and $b n d_{l o}$ and $b n d_{u p}$ are the lower and the upper boundaries. That means: if calf is narrower than the lower boundary, the estimated fit is too wide (-2) and vice versa.

The fit estimation model (the boundaries) is expressed in a very simple form as a linear function of the measured circumference as follows:

and

$$
\begin{aligned}
& \operatorname{bnd}_{l o}=k_{l o} * \operatorname{circ}+n_{l o} \\
& \operatorname{bnd}_{h i}=k_{h i} * \operatorname{circ}+n_{h i}
\end{aligned}
$$

In this equation we rely only on the boot measurements $(\operatorname{circ})$. The parameters $k_{l o}, n_{l o}, k_{h i}, n_{h i}$ are unknown and have to be defined using optimization. In the optimization process we search for such values of the parameters that result in maximization of the estimation accuracy function. Since the model is extremely simple we do not have the problem of overfitting, therefore, all training data may be used in the optimization.

The final estimation model is enhanced with some additional information. However, this is out of scope of this paper.

The estimation accuracy of the final estimation model is $71.3 \%$. As a matter of fact, in $85 \%$ subjects would select the right fitting boot when relying on our UCS BoC system. That means when BoC estimation model estimate a boot as acceptable, then a subject would buy it in $85 \%$.

\section{Conclusions}

In this work we introduced a system for estimating the fit of boots in calves. The system consists of the measurement device and estimation model. It is a self-standing solution for a boot reseller, however, it work best in the combination with the lower shoe recommendation system (see [6]).

We have shown several advantages of our system over the existing ones for boot selection assistance. The customer does not need any knowledge about boot standards, complex rules are avoided, the boots are not classified in limited number of badly defined classes etc. Here, the boots are measured and the measurement is stored in a database. And the system makes a decision for a customer.

The final result shows that the boots proposed by UCS BoC system satisfies the customer in more than $85 \%$. In comparison with random boot selection (i.e. without knowledge about the shoe width) this is a big improvement that will decrease return rates of purchased boots and increase the online sale of boots.

\section{References}

1. US Army anthropomorphic measurement campaign (accessed 2011): www.dtic.mil/dticasd/docs-a/anthro military.html

2. Duo boots web shop (accessed 2012): www.duoboots.com

3. Wide widths web shop (accessed 2012): www.widewidths.com

4. Simply be web shop (accessed 2012): www.simplybe.co.uk,

5. Viva la diva web shop (accessed 2012): http://www.vivaladiva.com/shop/page?pageld=3353

6. Omrčen, D., Jurca, A. (2011): "Shoe size recommendation system based on shoe inner dimension measurement", Proc. of 3D Body Scanning Technologies, Lugano, Switzerland, 2011

7. Russell, S., Norvig, P., "Artificial Intelligence: A modern approach", 2nd edition, Pearson Education, 2003 